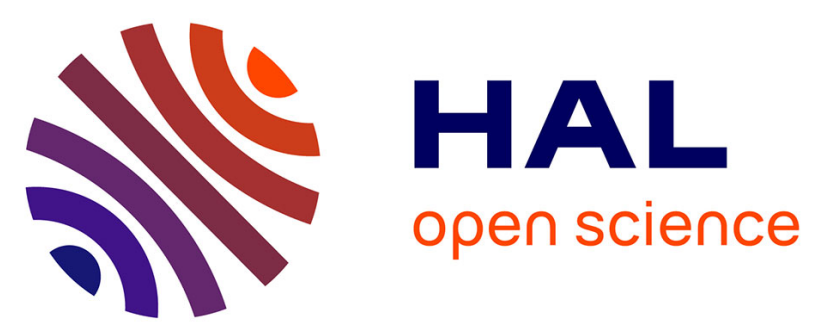

\title{
A User-Centered Approach to Adapt the Human-Machine Cooperation Strategy in Autonomous Driving
}

Stéphanie Coeugnet, Franck Mars, Mercedes Bueno, Chouki Sentouh, Jean-Christophe Popieul, Arnaud Koustanai, Annie Pauzie, Hélène Tattegrain

\section{To cite this version:}

Stéphanie Coeugnet, Franck Mars, Mercedes Bueno, Chouki Sentouh, Jean-Christophe Popieul, et al.. A User-Centered Approach to Adapt the Human-Machine Cooperation Strategy in Autonomous Driving. 21st Congress of the International Ergonomics Association (IEA 2021), Jun 2021, VANCOUVER, Canada. pp. 600-606, 10.1007/978-3-030-74608-7_73 . hal-03228345

\section{HAL Id: hal-03228345 \\ https://hal.science/hal-03228345}

Submitted on 18 May 2021

HAL is a multi-disciplinary open access archive for the deposit and dissemination of scientific research documents, whether they are published or not. The documents may come from teaching and research institutions in France or abroad, or from public or private research centers.
L'archive ouverte pluridisciplinaire HAL, est destinée au dépôt et à la diffusion de documents scientifiques de niveau recherche, publiés ou non, émanant des établissements d'enseignement et de recherche français ou étrangers, des laboratoires publics ou privés. 
This document is a preprint version of the following paper:

Coeugnet, S., Mars, F., Bueno, M., Sentouh, C., Popieul, J.-C., Koustanaï, A., Pauzié, A., Tattegrain, H. (2021). A user-centered approach to adapt the human-machine cooperation strategy in autonomous driving. In: Black N.L., Neumann W.P., Noy I. (eds) Proceedings of the 21st Congress of the International Ergonomics Association (IEA 2021). Lecture Notes in Networks and Systems, vol 221, pp 600-606. Springer, Cham. doi:10.1007/978-3-030-74608-7_73

\title{
A user-centered approach to adapt the human-machine cooperation strategy in autonomous driving
}

\author{
Stéphanie Coeugnet ${ }^{1}$, Franck Mars ${ }^{2}$, Mercedes Bueno ${ }^{1}$, Chouki Sentouh ${ }^{3}$, Jean- \\ Christophe Popieul $^{3}$, Arnaud Koustanaï ${ }^{4}$, Annie Pauzié ${ }^{5}$ and Hélène Tattegrain ${ }^{5}$ \\ ${ }^{1}$ VEDECOM Institute, 78000 Versailles, FRANCE \\ stephanie.coeugnet-chevrier@vedecom. fr \\ ${ }^{2}$ Université de Nantes, Centrale Nantes, CNRS, LS2N, 44000 Nantes, FRANCE \\ ${ }^{3}$ LAMIH, UPHF, 59300 Famars, FRANCE \\ ${ }^{4}$ Laboratory of Accidentology, Biomechanics and Driver Behavior, 92000 Nanterre, FRANCE \\ ${ }^{5}$ Gustave Eiffel University, LESCOT, 69500 Bron, FRANCE
}

\begin{abstract}
Using an integrative user-centered approach, the research project aimed at designing and assessing an adaptive HMI to improve the safety and the quality of the take-over in a level-3 automated vehicle.

Future autonomous driving users confirmed the need for the monitoring systems to improve safety as long as they are simple and not intrusive. They request specific information to understand and initiate appropriated actions in case of a critical situation. However, to avoid overloading and detrimental effects, it is important that the system limits the amount of information at the right moment.
\end{abstract}

Keywords: autonomous driving, driver monitoring, HMI, adaptive system

\section{Introduction}

The autonomous vehicle should increase driving comfort and road safety [1]. In this way, level-3 SAE systems should allow the driver to cede full control of all safetycritical function under specific conditions [2]. However, the driver is intended to respond appropriately to a request to intervene as a fallback to perform the dynamic driving task. Given this requirement to re-engage in the vehicle control loop and limitation that driver has in the monitoring road environment, a key issue is how to design time, modality, and frequency of transfer of control requests [3].

Studies on partial and conditional automation have already shown that an incorrect understanding of the system's current actions can lead to inappropriate or unsafe drivers' behaviors [4]. The profound change in driver activity brought about by automation (i.e., supervision, management of transitions, management of conflicts of intent) will require the design of new modalities of interaction with the vehicle. Designing a better dialogue between the driver and the vehicle will be essential to guarantee the acceptance, safety, and more generally, the quality of use of the autonomous vehicle [5]. To this end, the driver needs to receive information about the system's functioning and limits (e.g., the period of autonomous mode). Similarly, the vehicle needs to re- 
ceive some information about the driver's state using an integrative monitoring system. The research project within the framework of which the work reported in this paper was carried out sought to respond to these issues with a multidimensional approach to ergonomics. The overall objective was to adapt the HMI of a level 3 automated vehicle to the driver's state. The initial steps of the project consisted in a series of studies that will be briefly outlined here. In a first objective, six focus groups completed large-scale questionnaires involving 2619 participants. The results showed that the higher the drivers' acceptance of automated vehicles, the more they reported trust in the high automation levels. The results also highlighted an essential need for a progressive level of information in the case of a planned take-over and the guidance of the relevant actions to be taken in unplanned take-over using visual and auditory information [6]. The driver profiles analysis, including age and driving experience, was conducted and showed a need for an advanced HMI according to the skill driving level and use time. Then, in a second objective, a set of three driving simulator studies investigated specific aspects of driver monitoring (i.e., visual, postural, and physiological indicators). The visual behavior study used partial-least-square regressions to model drivers' visual strategies during autonomous driving to determine the characteristic patterns of an out-of-the-loop driver. The results showed that beyond the time spent looking at areas of interest crucial for maintaining situational awareness (road center, adjacent traffic lane, left rear-view mirror, in particular), it is necessary to consider indicators of gaze dynamics (transitions between areas) to obtain the most predictive models [7]. Postural studies have tested the position of the joint centers and the distribution of contact pressure in order to define the best postural indicator for monitoring. The results showed that pressure measurements can be used to extract relevant parameters to detect changes in posture [8]. The study on the internal state of the driver aimed to highlight physiological indicators of the driver's attentional state as a function of cognitive distraction induced by increased cognitive load or sadness. The results showed that it was possible to differentiate the driver's activity and emotional state with different physiological measurements: cardiac variability to detect emotion, breathing amplitude to distinguish the type of activity of the driver in a state of sadness, and the breathing period to differentiate activity in a neutral emotional state [9]. This paper follows the conclusions of the previous studies, starting with the objective to develop a global diagnostic of the driver state based on the three types of indicators (i.e., gaze, postural, and physiological indicators). In parallel, a new HMI has been designed taking into account the recommendations of the focus group study. The HMI and the driver monitoring system were implemented on a Wizard of $\mathrm{Oz}$ (WOz) vehicle (active commands managed by a professional driver without awareness of the driver). In addition to those developments, this paper also presents the preliminary results of a final study using this vehicle on open roads, which was performed to assess both the impact and the acceptance of the system. 


\section{Indicators and diagnose of the driver's state}

The implementation of several treatments allowed us to identify the state graph management of the vehicle that controls the different modes of automation. Initially, we designed the signals of the various sensors used to measure the driver's state and the vehicle's actions:

- the visual activity monitoring system, which provided diagnostics on the collection of perceptive information in the road scene from eye-tracking data,

- the postural monitoring system that provided diagnostics of the driver's posture, hand and foot positions from camera data,

- the physiological monitoring system that provided diagnostics on the attentional level of the driver from cardiac, respiratory, and electrodermal data,

- the system for reporting information on driving actions on the vehicle.

The Fig. 1 shows the processes of the diagnostics fusion and the related monitoring functions.

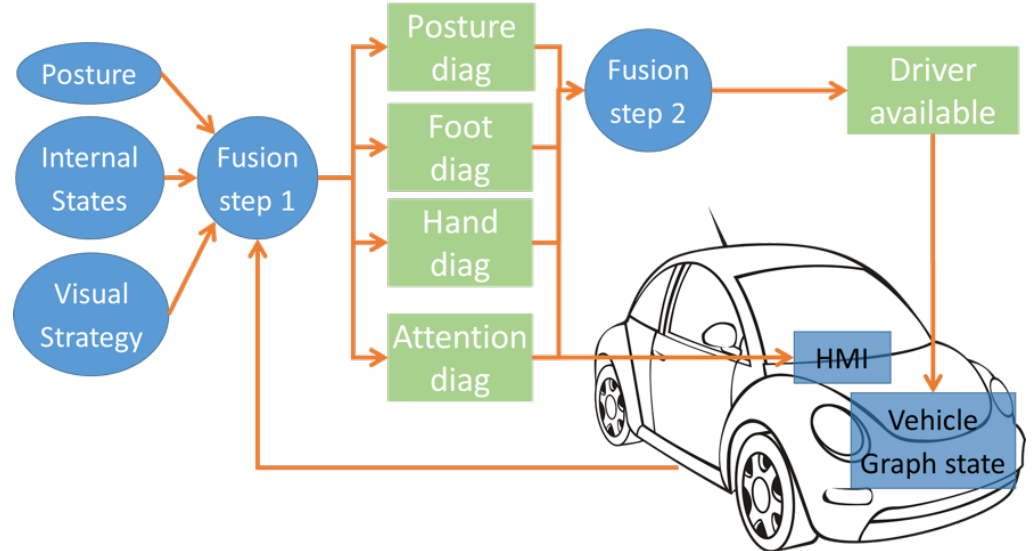

Fig. 1. Organization of monitoring and fusion processes.

From these indicators, the first step of merging the data using decision tables to create three diagnostics indicated whether the conditions on the body's positions, hands, and feet were correct. Finally, a fourth diagnosis considered that the attentional level was sufficient by exploiting the diagnoses of visual strategies and internal states. The second phase of fusion used these diagnostics to define a global diagnosis of the driver's ability to regain vehicle control.

\section{Design of the HMI}

The designed HMI served and communicated to the driver information related to both the driving situation and the autonomous driving system's operation. The objective was to warn the driver in the case of a potentially dangerous condition detection such as drowsiness or distraction, especially when taking back control. When returning to 
manual driving, when requested by the system, the HMI's role was to highlight the contextual elements that the driver must consider in order to regain control safely:

- Entry into delegation: before activating automated driving, the HMI allowed the driver to check that the system correctly perceived the driving environment (e.g., lanes, traffic). It supported the transition between the driver and the autonomous driving system by clearly indicating authority transfer.

- Road monitoring during automated driving : the HMI displayed the elements of the driving scene analyzed by the system (e.g., headway distance), thus allowing the driver not only to check the operation of the system but also to be able to make an immediate diagnosis of the driving situation and anticipate a possible need for planned or unplanned take-over request.

- Control resumption by the driver: during planned control recovery, the HMI informed the driver in advance, allowing him/her to prepare appropriately. During unplanned (sudden) take-over request, the HMI warned the driver with an adequate level of importance, indicating the situation to be managed imminently (identification of critical events).

The Fig. 2 presents the final HMI.

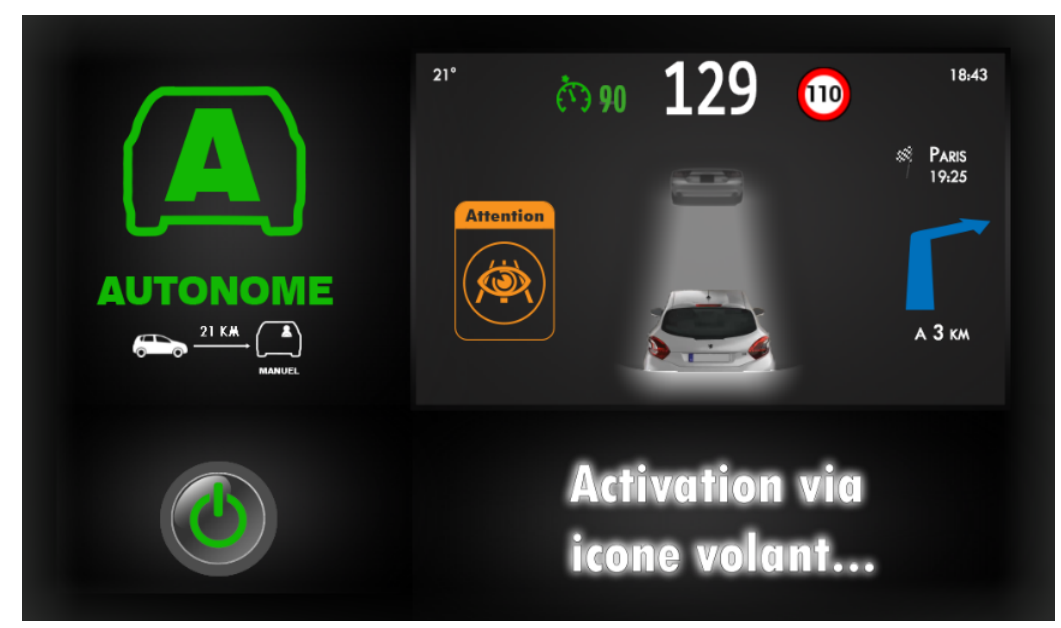

Fig. 2. Final designed HMI.

The HMI specifications were defined using the "five W method": What, Where, hoW, When and Who. Regarding the type of information to be displayed on the interface (What to display?), five groups were defined: the activation command (button), the driving mode indicator, the information contributing to a good "situational awareness", GPS navigation, and alert messages. To facilitate the understanding and urgency of the messages by the driver, a color code was defined. The fifth question, "Who?", aimed to identify the conditions for the HMI content evolution. The driver monitoring module diagnosed the driver's condition based on indicators related to his/her visual strategies, posture, and internal state described above. It also identified the driver's actions (e.g., resume a driving posture by putting the hands on the steering 
wheel and bringing the feet to the pedals). To do this, the driver monitoring manager interacted with the HMI manager module to request the display of visual messages on the screen and/or the broadcasting of voice messages. The HMI module was also in charge of not overloading the driver by managing the sequencing of visual or audio messages.

\section{Experimental assessment of the system - a WOz study}

\subsection{Method}

Fifty-two drivers $(26$ men, mean age $=38.5$ years $)$ participated in the WOz study on public roads. The $\mathrm{WOz}$ vehicle could be driven by the copilot (or wizard) with a joystick hidden to the participants, thus simulating the level-3 automated driving. A simplified version of the HMI presented in the previous section was used in this study. Specifically, the HMI provided about the activation command (button), the driving modes (manual driving, automated driving), GPS navigation, and alert messages (take-over requests). During the automated driving, participants were able to engage in non-driving related activities (e.g., reading, playing a game on a tablet), and they received two types of take-over requests: unplanned (budget time $=8 \mathrm{sec}$ ) and planned (budget time $=45 \mathrm{sec}$ ). Drivers' visual behavior, posture, and physiological measures (heart rate, skin conductance, respiration), were recorded using the SmartEye Pro dx remote eye-tracking system, two Time of flight cameras (PMD Pico Monstar and Softkinetic DepthSense DS325), and the BIOPAC acquisition system, respectively. The information about the driver state was processed offline. At the end of the drive, a semi-directive interview was conducted to explore drivers' opinions following the automated driving and their expectations about the monitoring system's functioning (only the results related to the monitoring will be presented in this article).

\subsection{Results}

Analysis of interview data revealed that almost all participants (97.7\%) considered that the system should assist drivers depending on their state. Most of them mentioned that it would be useful in case of drowsiness, annoying or lengthy trips (see Fig. 3).

Participants themselves were willing to be monitored with sensors $(86.1 \%)$, but the system must be more simple, less intrusive, and less restrictive to be accepted (e.g., bracelet or sensors integrated into the steering wheel). 57.1\% considered that the monitoring system would be relevant during the whole driving for security reasons and more reassurance. However, $40.5 \%$ would prefer to be assisted only during more complex contexts (e.g., urban driving) or specific situations that may affect their vigilance (e.g., highway). 


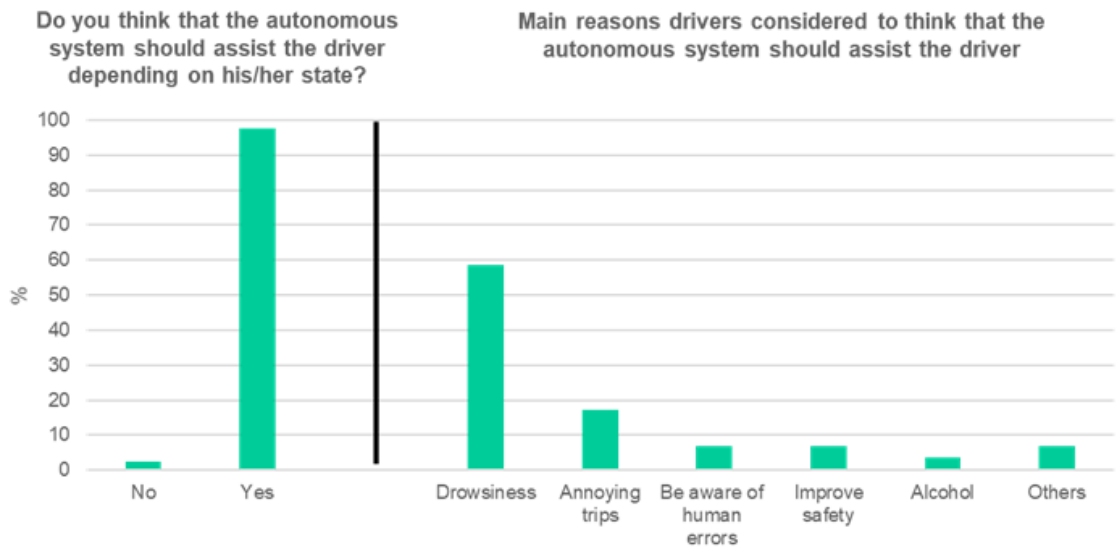

Fig. 3. Participants' opinion about the assistance of the autonomous system depending on the driver state.

Concerning the monitoring feedback, the visual and auditory combination was elicited by $44 \%$ of the participants. Most of them signaled that the visual modality would not be useful by itself in case of drowsiness or inattention to the HMI. However, $27.9 \%$ of the participants preferred the single visual mode because they considered it less aggressive and less disturbing to the other passengers (e.g., sleeping children). Besides, they were asked about their preferences regarding the pictogram type associated with the driver's state. They were shown and could decide between a general red pictogram ("improper driver state", see Fig. 4a) or specific red pictogram informing about an improper posture (Fig. 4b), hands-off (Fig. 4c), foot-off (Fig. 4d), eyes-off (Fig. 4e), and drowsiness (Fig. 4f).

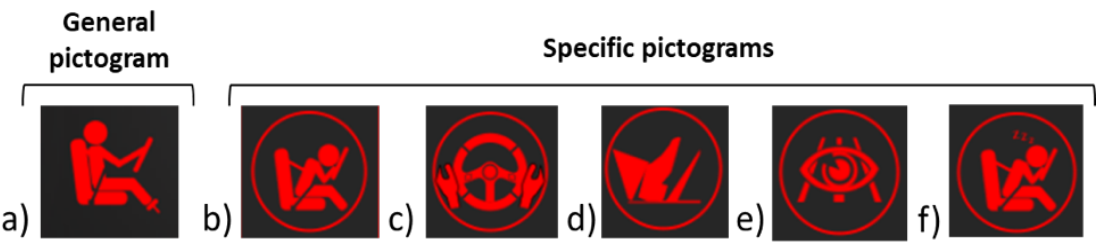

Fig. 4. General and specific pictograms about driver state.

More than half of the participants $(61.9 \%)$ preferred the specific pictograms to be aware of the problem and correct it. However, some of them were concerned about receiving warnings too frequently, and others indicated that only the drowsiness pictogram would be relevant. Participants opting for the general pictogram cited that specific pictograms would involve too much information to process, which could be irritating. 


\section{Discussion}

Based on the users' needs studies and the final assessments, main implications emerged from this research project: (1) monitoring systems should be favored - or even integrated systematically - in vehicles with automated driving ; (2) the presence of driver monitoring systems must be justified by a significant gain in terms of comfort and safety; (3) a positive point of view of autonomous vehicles improve the acceptance of the monitoring and its related HMI. Using a user-centered design approach helped identify and consider the most relevant future autonomous vehicles' expectations and needs. This approach could be used to design monitoring and cooperative systems. Carrying out experiments on the road with potential users of automated driving systems and implementing suitable methodologies constituted a significant advance proposed by this research project. These assessments lead to the safer and more efficient design of the Level 3 autonomous vehicle. Future users seem convinced of the need for the monitoring systems as useful aids to improve safety. However, to be accepted, it is necessary that these systems are integrated into the vehicle and easy to use. In case of critical situations, the system should provide specific information about the cause of the problem. Nevertheless, it is important to find an optimal balance between the frequency and the amount of information provided to the system to avoid it from being counterproductive.

\section{Acknowledgment}

This research project (AUTOCONDUCT - ANR-16-CE22-0007) was supported by grants from the French National Research Agency (ANR - Agence Nationale pour la Recherche). The authors are grateful to the different study participants for their commitment, interest, and cooperation.

\section{References}

1. Reimer. B. (2014). Driver assistance systems and the transition to automated vehicles: a path to increase older adult safety and mobility? Public Policy \& Aging Report, 24 (1), 27 31.

2. SAE International (2018). Taxonomy and definitions for terms related to driving automation systems for on-road motor vehicles, SAE Standard J3016, Report No. J3016-201806, Warrendale, PA.

3. Roche F, Somieski A, Brandenburg S. (2019). Behavioral Changes to Repeated Takeovers in Highly Automated Driving: Effects of the Takeover-Request Design and the Nondriving-Related Task Modality. Human Factors, 61(5), 839-849.

4. Carsten, O., Martens, M.H. (2019). How can humans understand their automated cars? HMI principles, problems and solutions. Cognition Technology \& Work, 21, 3-20.

5. Hartwich, F., Beggiato, M., \& Krems, J. F. (2018). Driving comfort, enjoyment and acceptance of automated driving-effects of drivers' age and driving style familiarity. Ergonomics, 61(8), 1017-1032. 
6. Bel, M., Pauzié, A., Fehat, L., Kraiem, S. (2019). Needs analysis, design assistance and acceptability of the automated vehicle. EPIQUE, Ergonomics Psychology Conference, Lyon, France.

7. Schnebelen, D., Charron, C. \& Mars, F. (2020). Predicting the out-of-the-loop phenomenon from visual strategies during highly automated driving. Accident Analysis \& Prevention, 148, 105776.

8. Zhao, M., Beurier, G., Wang, H., and Wang, X. (2018). In Vehicle Diver Postural Monitoring using a Depth Camera Kinect, SAE Technical Paper 2018-01-0505, 2018, doi:10.4271/2018-01-0505.

9. Hidalgo-Muñoz, A., Jallais, C., Evennou, M., Ndiaye, D., Moreau, F., Ranchet, M., Derollepot, R., Fort, A. (2019). Hemodynamic responses to visual cues during attentive listening in autonomous versus manual simulated driving: A pilot study. Brain and Cognition, 135. 